\title{
Chances for Earth-Like Planets and Life Around Metal-Poor Stars
}

\author{
Hans Zinnecker \\ Astrophysikalisches Institut Potsdam, An der Sternwarte 16, \\ 14482 Potsdam, Germany
}

\begin{abstract}
We discuss the difficulties of forming earth-like planets in metal-poor environments, such as those prevailing in the Galactic halo (Pop II), the Magellanic Clouds, and the early universe. We suggest that, with fewer heavy elements available, terrestrial planets will be smaller size and lower mass than in our solar system (solar metallicity). Such planets may not be able to sustain life as we know it. Therefore, the chances of very old lifeforms in the universe are slim, and a threshold metallicty (90\% solar?) may exist for life to originate on large enough earth-like planets.
\end{abstract}

\section{Introduction}

We do not know whether life is widespread in the Galaxy and the universe. Many factors have to conspire to form habitable earth-like planets, with the right conditions to sustain a biosphere (see the book "Rare Earth" by Ward and Brownlee, 2000). One of these factors seems to be a certain amount of heavy elements and metals ( $\mathrm{C}, \mathrm{N}, \mathrm{O} ; \mathrm{Mg}, \mathrm{Si}, \mathrm{Fe}$; radioactive elements and also phosphor central to the RNA/DNA world). Therefore the question arises if there is a critical metallicity that has to be reached before life can originate. For example, life-bearing planets probably need enough liquid water (containing oxygen) at their surface and a sufficiently strong magnetic field generated in their iron core to shield any incipient lifeforms from lethal external UV radiation and energetic cosmic rays. Both the amount of oxygen (to form water) and the amount of iron (to form a sizable core) are likely to be significantly reduced in a metalpoor galactic environment, such as in the Galactic halo or in globular clusters. We refer to these conditions as Population II conditions, but broaden them to include star and planet formation environments in the Magellanic Clouds, with heavy element abundances between $1 / 4$ solar (LMC) to $1 / 10$ solar (SMC).

In this short note, we argue that earth-like planets (i.e., between 0.5 and 2 earth masses) are unlikely to form under conditions as metal-poor as the SMC or even the LMC (regions with dust to gas mass ratios as low as 1:1000 or $1: 2000$ ), because with such a reduced dust content any terrestrial planets that may form in circumstellar disks around solar-mass stars (Beckwith \& Sargent 1996) will be significantly smaller in size than Earth (perhaps Mercury or Mars 
size), and hence unable to retain enough of an atmosphere, among other things. Thus we suspect that there is nobody up there in the Galactic halo or in the Magellanic Clouds looking down on us in the Galactic disk!

\section{Metallicity Dependence for Earth-like Planet Formation}

The Earth has been formed by the collisions of some 10 Mars-sized protoplanets, which themselves have been formed through runaway and oligarchic growth of so-called planetesimals (solid bodies with sizes of order of $5 \mathrm{~km}$ and masses of the order of $10^{18} \mathrm{~g}$ ); see Kokubo \& Ida (2000) for runaway and oligarchic growth and Hayashi et al. (1985) for the initial masses of planetesimals at an orbital radius of $1 \mathrm{AU}$. If we now make the assumption that in a metal-poor environment the dust mass density scales linearly with the metallicity (a reasonable assumption it seems, see Bouchet et al. 1985), we can extrapolate the formalism of Kokubo \& Ida (2000) to the case of a metal-poor circumstellar disk with a reduced dust surface mass density, proportional to metallicity $\left(Z / Z_{\odot}\right)$. The result is that the final masses of protoplanets for $1 / 4$ and $1 / 10$ solar metallicity will be scaled down to 0.02 earth masses and 0.005 earth masses, respectively (instead of 0.16 earth masses for solar metallicity), as the mass of a protoplanet $\left(M_{\text {proto }}\right)$ scales with the $3 / 2$ power of the dust surface mass density $(\Sigma)$ :

$$
M_{\text {proto }} \sim \Sigma^{3 / 2} \sim\left(Z / Z_{\odot}\right)^{3 / 2}
$$

The mass of a protoplanet after oligarchic growth and in a circular orbit is essentially the integrated surface mass density of dust in a ring whose width $(w)$ is given by the Hill (Roche) criterion:

$$
w \approx 10\left(\frac{M_{\text {proto }}}{M_{*}}\right)^{1 / 3} a
$$

where $M_{*}$ is the mass of the central star $\left(1 \mathrm{M}_{\odot}\right)$ and $a$ is the orbital radius (1 AU); see Kokubo \& Ida (2000), see also Kokubo's (2001) review.

We find $w \approx 0.1 A U$ for $Z / Z_{\odot}=0.1$. It follows that in the $1 / 4$ or $1 / 10$ solar metallicity cases the orbital spacing of protoplanets is twice or three times as tight as in the solar metallicity case. The growth timescale for protoplanets at $a=1 \mathrm{AU}$ will be about 100000 to $250000 \mathrm{yr}$ in the two metal-poor cases, inversely proportional to the surface mass density, i.e., the metallicity. The protoplanet system formed by oligarchic growth will become orbitally unstable on longer timescales (eccentricity pumping and orbit crossing) and will form more massive bodies by collisional accretion. How massive will the most massive terrestrial planet be (the equivalent of the Earth)? Long-term N-body simulations (such as those by Wetherill \& Stewart 1993) would need to be carried out, but we can estimate the outcome. We need 5-20 sticky collisions (ignoring fragmentation) to grow the protoplanets to the size and mass of Mars (10\% earth mass). The collision time between the protoplanets turns out to be of the order of a few times $10^{8}$ years, assuming a velocity dispersion of the order of the orbital velocity $\left(30 \mathrm{~km} \mathrm{~s}^{-1}\right)$ and a geometric cross-section for collisions (protoplanet sizes of order $1000 \mathrm{~km}$ ). This implies these protoplanets may just make it into Mars-like objects in half a Hubble time, but they won't make it into Earth-sized planets; 
there is not enough time and there is not enough material within the effective annular region for bigger planets to form. The above non-linear dependence of the mass of a protoplanet on metallicity suggests that we need a threshold metallicity of very close $(90 \%$ ?) to solar to obtain an earth-like planet and a planetary system similar to our own.

\section{A Threshold Metallicity for Life to Begin?}

In the previous section, we have found that terrestrial planet formation is a sensitive function of metallicity. It seems we need a metallicity rather close to solar (at least half solar) for planets the size and mass of Earth to form. This is due to the non-linear dependence of mass growth and timescales on the surface mass density of dust in the habitable zone (near $1 \mathrm{AU}$ ). Decreasing the surface mass density of solid material has a dramatic effect on the final outcome: rather than Earth-like planets we get asteroids and gravel, not suitable for life.

Life depends in many ways on the size and mass of a planet. Firstly, as mentioned before, mass $M$ and radius $R$ determine the gravity of planet ( $\left.g=G M / R^{2}\right)$ which grows roughly proportional to size. Therefore bigger planets can better retain more of an atmosphere, which is crucial for our life (oxygen, ozone, carbon dioxide, water and rain), and may be crucial for other life-forms, too. Secondly, if too small, a planet will not have enough of an iron core to generate a substantial magnetic field by the dynamo effect, which is required to shield the surface from cosmic ray bombardment. Thirdly, if the planet is too small, there is not enough heat generated by the radioactive elements and by the collisional build-up that the planet can sustain volcanic activity and plate tectonics for a long time, both instrumental for the carbondioxide cycle and a stable greenhouse effect. In short, if the planet is too small, it cools off quickly and becomes a dead world.

So far we have not discussed the issue of the formation of a Jupiter-like planet under metal-poor conditions. We know that the presence of Jupiter in the solar system is a big advantage for protecting the earth from late impacts. If Jupiter could not form under metal-poor conditions, because the seed rocky core does not grow large enough to attract and to accrete the gas (Mizuno 1980), we may not have the benign environment and shelter that enables life to blossom - in case low-metallicity conditions somehow manage to produce an Earth after all.

Finally, we don't know at this point, what would be the the mix of chemical elements on a metal-poor (Pop II) planet. Perhaps gas phase studies of the Magellanic Clouds could help. In any case, oxygen, nitrogen, carbon, and sulphur are needed to make amino-acids, the basic ingredients for life to start. Liquid water with all its wonderful properties is also needed to get going. Will there be relatively less water on a planet that started out with metal-poor initial conditions? Less rain? Will the chemistry and mineralogy develop differently? These are interesting questions to study in the future. Today there are at least some indications that the bulk of solar-type stars with giant planets seems to be at least as metal-rich as the Sun (Gonzales et al. 2001; Santos et al. 2001). 


\section{Caveats and Observational Tests}

We note that the metallicity bias against Earth-like planet formation can be circumvented if circumstellar disks around Pop II stars are correspondingly smaller at lower metallicity (smaller implies lower disk angular momentum). In this case the smaller dust-to-gas mass ratio is compensated by distributing the dust mass over a smaller disk, thus keeping dust surface mass density as high as in the solar metallicity case. [It is the dust surface mass density that enters all the equations and causes the diversity of planetary systems (cf., Kornet et al. 2001)]. By squeezing the disk (by a factor of 2-3 for the LMC and SMC metallicity conditions), Earth-like planets around metal-poor stars should be able to form. Perhaps Jupiter-like planets, too, although by a different process: namely by direct gravitational instability of a gas disk, a process which is independent of metallicity and the dust content of a protostellar disk (Boss 2002). However, at present there is no reason to assume that metal-poor gaseous systems are low angular momentum systems, (for example, the Pop II wide binary frequency seems to be similar to that of Pop I stars, see Allen et al. 2000 and references therein).

In above context, the search for Jupiter-like planets around thousands of stars of the Pop II Galactic globular cluster Omega Cen may be particularly worthwhile, as this cluster unlike any other globular cluster contains stars with a range of metallicities, thus allowing a new test of the metallicity dependence of planet formation (K. Freeman, personal communication). The Omega Cen cluster ( $1 / 30$ solar in the mean) is a better choice than the much denser, constant metallicity ( $1 / 5$ solar) 47 Tuc cluster, where such a search was unsuccessful (Gilliland et al. 2000) and where theoretical expectations were low anyway due to the high stellar density in the cluster (Bonnell et al. 2001).

We also note that, although there are no very old, metal-poor Pop II $1 \mathrm{M}_{\odot}$ stars on the Main Sequence any more, many slightly lower-mass Main Sequence stars $\left(0.8 \mathrm{M}_{\odot}\right)$ of old age and low-metallicity composition exist in globular clusters (see Baraffe et al. 1997 for models of metal-poor, low-mass stars). Pop II stars of mass below $0.8 \mathrm{M}_{\odot}$ have not yet evolved to red giants and thus would not yet have swallowed their inner planets, should these exist (cf., Sackmann et al. 1993; Schröder et al. 2001). Therefore, life could in principle exist around these stars with stable conditions. Moreover, a metal-poor star of $0.8 \mathrm{M}_{\odot}$ and $Z=0.1 Z_{\odot}$ is about as bright as a solar metallicity star (cf. Baraffe et al. 1997, their Tables 2-5), and their habitable zones could be similar, with the caveat that the metal-poor star generates a lot more UV radiation than the solar-metallicity star. In conclusion: Stellar evolution would allow early Pop II lifeforms to survive, if they ever got off to a start. Thus in principle some life in the universe could be very old, if it has not destroyed itself.

\section{Life - Early on in the Cosmic History?}

How early in the cosmic history can earth-like planets form, how early can life originate? Lineweaver (2001) wrote an interesting article about the metallicity dependence of forming earth-like planets in the cosmos, retarding the onset of life. However, for simplicity he assumed a probability for Earth-like planet formation linearly proportional to metallicity above a certain threshold metallicity, 
which he guessed to be within a factor of two of $1 / 10$ solar. In this paper, we tried to quantify a threshold metallicity, and find a non-linear dependence of the protoplanetary mass on metallicity. The difference is that in our picture, no suitable planets form until a high degree of chemical enrichment has happened, while in Lineweaver's model earth-like planets can form in rather metal-poor gas, very early on in the cosmic history, albeit with a correspondingly small probability. We suggest his analysis should be redone including our refined calculation of a threshold metallicity. The difference is important, because if there is any chance for life to somehow start in the very early universe (i.e., in the first Gyr), it would have had some $12 \mathrm{Gyr}$ to evolve, which is 3 times more than life as we know it from planet Earth. Then the question "Where are they?" would be an even more serious one.

Acknowledgments. Thanks to M. Rozyczka and G. Wuchterl for helpful discussions. Thanks to MWFK Brandenburg for a travel grant (HSP-N programme). Thanks to Carol oliver and Ray Norris for bringing us all together on Hamilton Island. Thanks to M. Zadnik for being such a nice room-mate. Thanks to M. Burton for riding the jet-ski with me. Thanks to Ch. Lineweaver, F. Drake, and the referee for constructive comments. And thanks to R. D. Cannon for his post-Symposium hospitality in Sydney, including an excursion to Canberra (Mt. Stromlo Observatory).

\section{References}

Allen, C., Poveda, A., \& Herrera, M. A. 2000, A\&A, 356, 529

Baraffe, I., Chabrier, G., Allard, F., \& Hauschildt, P. H. 1997, A\&A, 327, 1054

Beckwith, S. V. W., \& Sargent, A. I. 1996, Nature, 383, 139

Bonnell, I. A., Smith, K.W., Davies, M. B., \& Horne, K. 2001, MNRAS, 322, 859

Boss, A.P. 2002, ApJ, 567, L149

Bouchet, P., et al. 1985, A\&A, 149, 330

Gilliland, R. L. et al. 2000, ApJ, 545, L47

Gonzalez, G., Laws, Ch., Tyagi, S., \& Reddy, B.E. 2001, AJ, 121, 432

Hayashi, C., Nakazawa, K., \& Nakagawa, Y. 1985, in Prototstars \& Planets II, ed. D. C. Black \& M. S. Mathews (Univ. of Arizona Press), 1100

Kokubo, E. 2001, Rev. Mod. Astron., 14, 117

Kokubo, E., \& Ida, S. 2000, Icarus, 143, 15

Kornet, K., Stepinski, T.F., \& Rozyczka, M. 2001, A\&A, 378, 180

Lineweaver, Ch. H. 2001, Icarus, 151, 307

Mizuno, H. 1980, Prog. Theor. Phys., 64, 544

Sackmann, I.-J., Boothroyd, A. J., \& Kraemer, K. E. 1993, ApJ, 418, 457

Santos, N. C., Israelian, G., \& Mayor, M. 2001, A\&A, 373, 1019

Schröder, K.-P. Smith, R. C., \& Apps, K. 2001, Astronomy and Geophysics, 42, 26

Ward, P. D., \& Brownlee, D. 2000, in Rare Earth, (New York: Springer)

Wetherill, G. W., \& Stewart, G. R. 1993, Icarus, 106, 190 


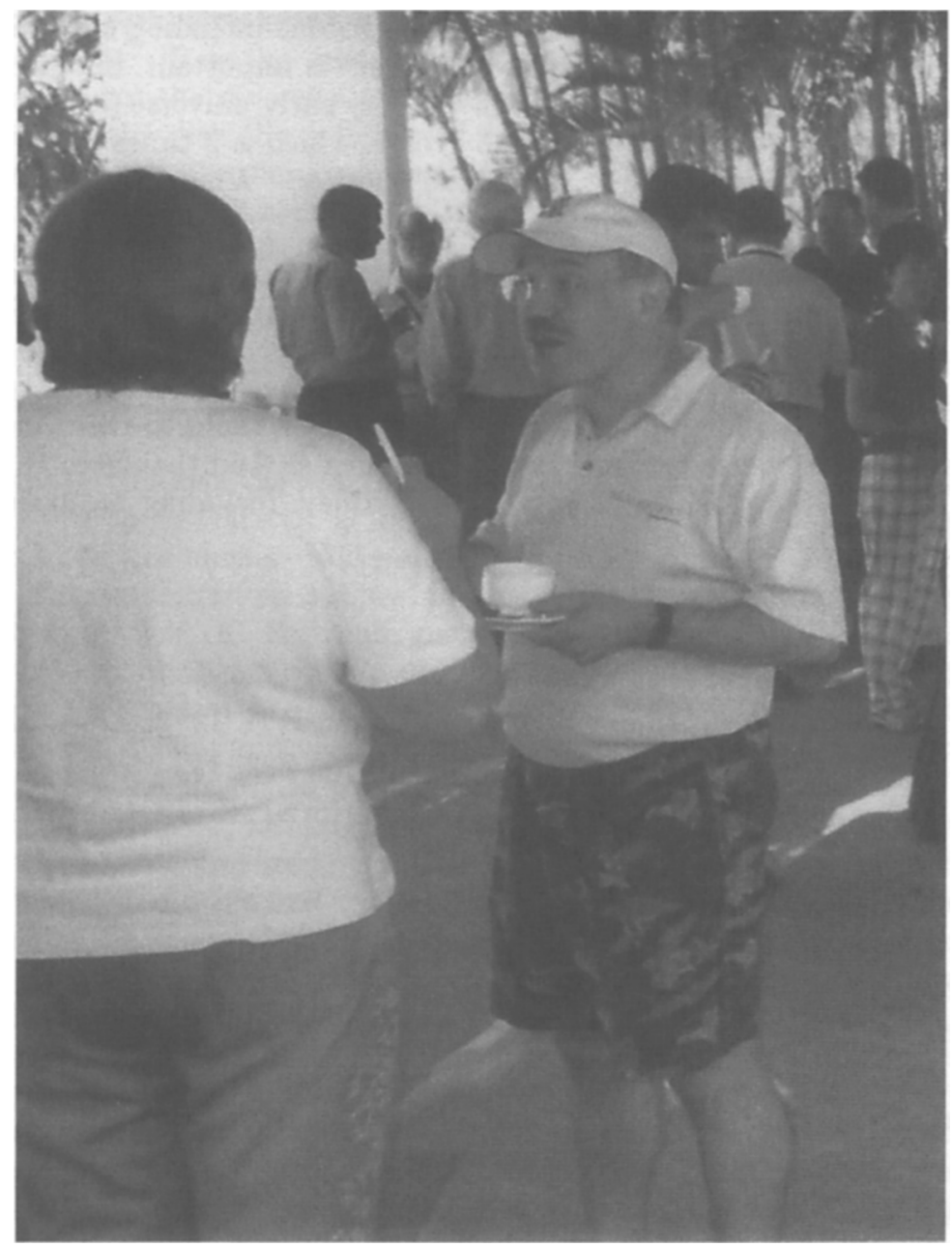

Hans Zinnecker (photo: Ulrich Dopatka) 